\title{
Separating the spectra of binary stars.*
}

\section{A simple method: Secondary reconstruction}

\author{
S. Ferluga ${ }^{1}$, L. Floreano ${ }^{2}$, U. Bravar ${ }^{3}$, and C. Bédalo ${ }^{1}$ \\ 1 Dept. of Astronomy, Univ. of Trieste - via G. B. Tiepolo 11, I-34131 Trieste, Italy \\ 2 INFN, Trieste - Padriciano 99, I-34012 Trieste, Italy \\ 3 Dept. of Physics, Univ. of Trieste - via A. Valerio 2, I-34127 Trieste, Italy.
}

Received October 10, 1995; accepted April 13, 1996

\begin{abstract}
We present a practical method for the analysis of spectroscopic binaries, reconstructing the lines of the two components of the system. We show that the problem of the separation of binary spectra can be solved in an easy way, under most common conditions.

One pair of observations may be sufficient, if taken at different orbital phases of the system, preferably at opposite quadratures. The separation procedure is discussed analytically, and a technique is described, which allows to restore the secondary lines in few steps. An algorithm is also provided, which derives the radial velocity of the secondary star, by directly analysing a difference line-profile obtained from the two input spectra.

The efficiency of the method is tested, by reconstructing artificial line-profiles and simulated binary spectra as well. Then the procedure is applied to the eclipsing binary $I Z$ Per, revealing for the first time its faint secondary spectrum.
\end{abstract}

Key words: methods: data analysis - binaries: spectroscopic — stars: individual: IZ Per

\section{Introduction}

A basic problem in the study of binary stars is that of separating the two original stellar spectra which are embedded in the composite spectrum of the system.

Going beyond the most trivial cases when both sets of lines were directly recognized by sight, early attempts

Send offprint requests to: Steno Ferluga (Internet e-mail: ferluga@ts.astro.it)

* Based on observations performed at the Observatoire de Haute Provence (OHP), and made available through the Trieste-Aurélie-Archive (TAA) trying to isolate the spectral contribution of each component date back to analogical techniques and hand-made computations (Wright 1954).

Various digital methods were later developed, aiming to separate the spectra of particular binaries with favourable orbital configurations, allowing a straightforward mathematical decomposition of the systemic spectrum.

\subsection{Occultation-based methods}

Totally-eclipsing binaries immediately make it possible to identify the spectrum of the uneclipsed component, so that the other can be simply obtained by a luminosityweighted subtraction and renormalization (Griffin R.\& R. 1986). The same approach has also been used in the absence of a real eclipse, by reproducing the known component with a simulated spectrum (synthetic or from a standard star), and then deriving the companion by difference.

It is the geometry of the system itself, that defines the mathematical operation required for spectral separation. For example, in the case of an atmospheric eclipse, as that of $\epsilon$ Aur, the spectrum of the obscure eclipsing body has been obtained as the ratio between the eclipsed and uneclipsed spectra of the system (Ferluga \& Mangiacapra 1991). Similarly, in $\zeta$ Aur-type binaries, the chromospheric spectrum of the supergiant can be extracted from limb-occultation observations, using a computational procedure involving spectra taken at different eclipse-phases (Griffin et al. 1993).

Partially-eclipsing binaries, at each phase of occultation, produce a composite spectrum which is given by a different linear combination of the two unknown spectra. This, in principle, should allow a complete spectral reconstruction, when starting from a suitable set of composite spectra, observed at different eclipse phases. Although 
conceptually simple, the implementation of this method has still to be fully developed.

\subsection{Doppler-shift methods}

The majority of spectroscopic binaries, being noneclipsing systems, require special separation methods, based on the different radial velocity of the components. In the simplest situations, the two spectra may consist of a few non-overlapping lines; this may happen for detached early-type binaries, observed at opposite elongation phases. In such conditions, the lines of each component may be isolated from the continuum by a practical divide-and-cut method (Pilachowski \& Sowell 1992).

Generally, however, more sophisticated separation and reconstruction techniques are required to treat the common cases when the two spectra are crowded with lines and very closely intertwined, and also when the Doppler splitting of input spectra is poor. This happens, for example, in the case of semidetached $(s d)$ and contact $(c)$ binaries, or when available observations concern only intermediate phases, instead of opposite elongations. Recently, different advanced methods have been independently presented.

Providing full spectral reconstruction under most general conditions, these new proposed methods are based on complex algorithms; they usually require a large number of composite spectra, observed at different rotational phases of the system, in order to extract the two components. In particular, the advanced disentangling method by Simon \& Sturm (1993) is based on the inversion of a linear-transformation matrix to separate the two components; its precision depends on the dimension of the matrix, which corresponds to the number of composite spectra employed. And also the sophisticated tomographic method by Bagnuolo \& Gies (1991), which is based on an iterative algorithm similar in principle to the tomography used in medical physics, has a precision depending on the number of composite spectra used in the reconstruction process.

\subsection{The present approach}

With the purpose of conceptual and practical simplicity, we developed an original Doppler-shift method to separate the spectra of binary stars. It requires only a pair of observations at different orbital phases (elongations preferably) and elementary computations.

A full reconstruction of the secondary absorption spectrum is obtained after few iterations, by applying a simple sequence of algebraic operations and Doppler-shift compensations to the pair of observed spectra. Then the primary spectrum is trivially derived by difference, renormalization and shift-compensation, from the systemic spectrum and the reconstructed secondary.
A larger number of available spectra, at various orbital phases, will obviously increase the signal-to-noise quality of the result; and this may be useful for faint targets. The procedure works powerfully even when the secondary spectrum is very weak, and/or when the primary and secondary lines overlap. In fact, the earliest conception of our method (Ferluga et al. 1991) was aimed precisely at the detection of faint double-lined eclipsing binaries.

\section{The reconstruction procedure}

Our separation method is based on the analysis of one pair of spectra of the system, obtained at two different orbital configurations. Both spectra must be taken out of eclipse or conjunction.

The method is applicable in most general cases (only secondary spectra with emission lines are excluded). We shall see that the extraction procedure requires, to converge, a secondary spectrum containing at least one window of flat continuum, in the observed spectral range.

\subsection{The input spectra}

Observations at opposite or near-opposite phases are preferable, and at quadratures (opposite elongations) are optimal. So the displacement of secondary lines is almost symmetrical in the two phases, and maximum at quadratures. Conventionally, for the two observed phases $\Phi_{1}$ and $\Phi_{2}$, we use the index 1 to label the phase with the most blue-shifted primary (most red-shifted secondary).

There are no special requirements for the adopted wavelength range $\left[\lambda^{\prime}, \lambda^{\prime \prime}\right]$, and for the spectral resolution, except that all instrumental settings must be the same for both spectra. Also the standard intensity-wavelength calibration, and the (optional) continuum-normalization as well, should be initially performed in an identical way.

The procedure is applicable, in principle, even if the input spectra have no recognizable continuum. Practically, in place of the continuum level, each input spectrum $\Phi_{\mathrm{i}}(\lambda)$ may be normalized with respect to its average intensity level $\int_{\lambda^{\prime}}^{\lambda^{\prime \prime}} \Phi_{\mathrm{i}}(\lambda) \mathrm{d} \lambda /\left(\lambda^{\prime \prime}-\lambda^{\prime}\right)$, coinciding with the continuum only if the integrated contribution of the lines is negligible. However, for simplicity's sake, we shall still use the word continuum instead of the expression average intensity, when describing normalization and renormalization procedures hereafter (e.g. in Sect. 2.4).

\subsection{The difference spectrum}

The first step of the procedure is to compensate for the radial velocity of the primary star in our intensitynormalized spectra. So we get two velocity-corrected spectra $C_{1}(\lambda)$ and $C_{2}(\lambda)$, where each primary line has its laboratory wavelength $\lambda_{\text {lab }}$.

The composite spectra $C_{1}(\lambda)$ and $C_{2}(\lambda)$ are then subtracted point-by-point one from the other: in this way the 
primary lines are eliminated (assuming the star is not intrinsically variable), and what we obtain is the difference spectrum

$$
S(\lambda)=C_{2}(\lambda)-C_{1}(\lambda)
$$

This special spectrum, which we shall also call $S$ spectrum, contains - in principle - no trace of primary lines (if the primary velocity has been correctly compensated for); while it shows symmetrical excursions around the zero level (that is now the continuum). Each secondary line is represented by an undulated feature, hereafter named $S$-profile, which consists of the line itself, flanked by (and possibly merged with) its capsized and shifted replication. Figure 1 shows how the $S$-spectrum is obtained by starting from two spectra observed at nearopposite phases.

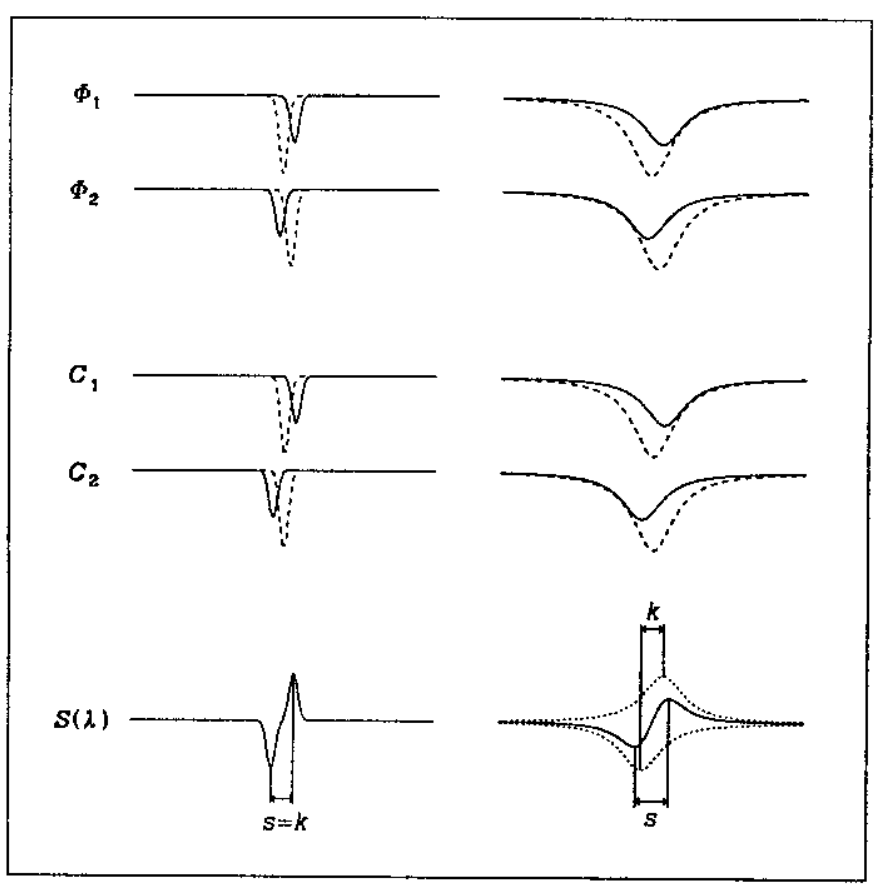

Fig. 1. Generation of the $S$-spectrum (schematic). Above: the input spectra $\Phi_{1}$ and $\Phi_{2}$, containing primary (dashed) and secondary (solid) features. Middle: the shifted spectra $C_{1}$ and $C_{2}$, both with the primary lines at rest-wavelength. Below: the difference spectrum $S=C_{2}-C_{1}$. Note that $s \neq k$ for merging profiles (right, dotted)

In practical applications, the secondary star is generally much fainter than the primary (up to only $1 \%$ ); so we shall realistically describe the secondary lines as an additional effect on the input spectra (though the treatment is independent of the secondary brightness). Then, if $F_{\mathrm{A}}$ is the spectrum of the primary star at rest (normalized to the continuum of the system), the contribution of the secondary star to the two input spectra can be represented with $f_{1}(\lambda)$ and $f_{2}(\lambda)$, defined by:

$$
C_{1}(\lambda)=F_{\mathrm{A}}(\lambda)+f_{1}(\lambda) ; \quad C_{2}(\lambda)=F_{\mathrm{A}}(\lambda)+f_{2}(\lambda) .
$$

Note that, while $F_{\mathrm{A}}$ is a normal spectrum (continuum=1), the functions $f_{1}$ and $f_{2}$ have the form of superimposed perturbations (continuum $=0$ ), to be renormalized later (Sect. 2.4). With the above definitions, the $S$-spectrum in Eq. (1) is reduced to:

$$
S(\lambda)=f_{2}(\lambda)-f_{1}(\lambda),
$$

where only the secondary signal is left (while the primary $F_{\mathrm{A}}$ is cleared off).

Since the twin images $f_{2}$ and $-f_{1}$ of the secondary lines (displaced and mirror-like) are entangled in the $S$ spectrum above, they should be first separated, re-oriented and shifted, and then recombined. We shall use an iterative method, restoring the line step by step, with an original shift-check-sum technique.

\subsection{Reconstruction of a line-profile}

We must state beforehand that, if the two images of a line are well separated within the $S$-profile, without overlapping, clearly each one is representative of the original feature. So, in this case, there is absolutely no problem.

Problems arise, instead, as soon as we want to recover the shape of a line when its original images partially overlap and neutralize each other, so that a merging $S$-profile is formed. It happens when the width of a line is large with respect to the velocity separation of input phases. In this case, profile restoration is needed.

Our reconstruction procedure starts from the pair of composite spectra $C_{1}$ and $C_{2}$, which through Eq. (1) provide the difference-spectrum $S(\lambda)$ to be processed. Our purpose is then to derive, in Eq. (2), the form of its constituent functions $f_{1}(\lambda)$ and $f_{2}(\lambda)$. They are symmetrical, because $f_{1}$ is a shifted replication of $f_{2}$. The Doppler shift, corresponding to the difference in radial velocity $\Delta v_{12}=v\left(f_{1}\right)-v\left(f_{2}\right)=v_{\mathrm{B}}\left(C_{1}\right)-v_{\mathrm{B}}\left(C_{2}\right)$ of the secondary component in the two spectra, will be hereafter indicated by $k=\lambda \cdot \Delta v_{12} / c$. So, it is identically:

$$
f_{2}(\lambda)=f_{1}(\lambda+k),
$$

with $k>0$ because of our initial choice of indexes.

Let us now assume for simplicity's sake a secondary spectrum having a single spectral line, and being equal to the continuum outside the interval containing that line. Supposing this interval is $\left[\lambda_{\mathrm{a}}, \lambda_{\mathrm{b}}\right]$ for $f_{2}(\lambda)$; then, according to Eq. (3), it will be $\left[\lambda_{\mathrm{a}}+k, \lambda_{\mathrm{b}}+k\right]$ for $f_{1}(\lambda)$.

It follows that a particular interval $\left[\lambda_{\mathrm{a}}, \lambda_{\mathrm{a}}+k\right]$ exists, in which $f_{2}(\lambda)$ displays the line-profile, while we still have $f_{1}(\lambda)=0$. Therefore, in this interval, from Eq. (2) we can derive $f_{2}(\lambda)=S(\lambda)$. So, in the interval $\left[\lambda_{\mathrm{a}}, \lambda_{\mathrm{a}}+k\right]$, both $f_{1}$ and $f_{2}$ are determined. 
Then, by knowing $f_{2}$ in the above interval, we are able to compute, from Eq. (3), the values of $f_{1}$ in the new interval $\left[\lambda_{\mathrm{a}}+k, \lambda_{\mathrm{a}}+2 k\right]$. Now, in this interval, from Eq. (2) we can also derive $f_{2}=S+f_{1}$. Thus, in the new interval $\left[\lambda_{\mathrm{a}}+k, \lambda_{\mathrm{a}}+2 k\right]$, both functions $f_{1}$ and $f_{2}$ can be obtained.

This leads through Eq. (3) to the values of $f_{1}$ in the next interval $\left[\lambda_{\mathrm{a}}+2 k, \lambda_{\mathrm{a}}+3 k\right]$, and so on. In such a way, by iteratively applying Eqs. (3) and (2), we are able to reconstruct the whole function $f_{1}(\lambda)$, representing the contribution of the secondary spectrum.

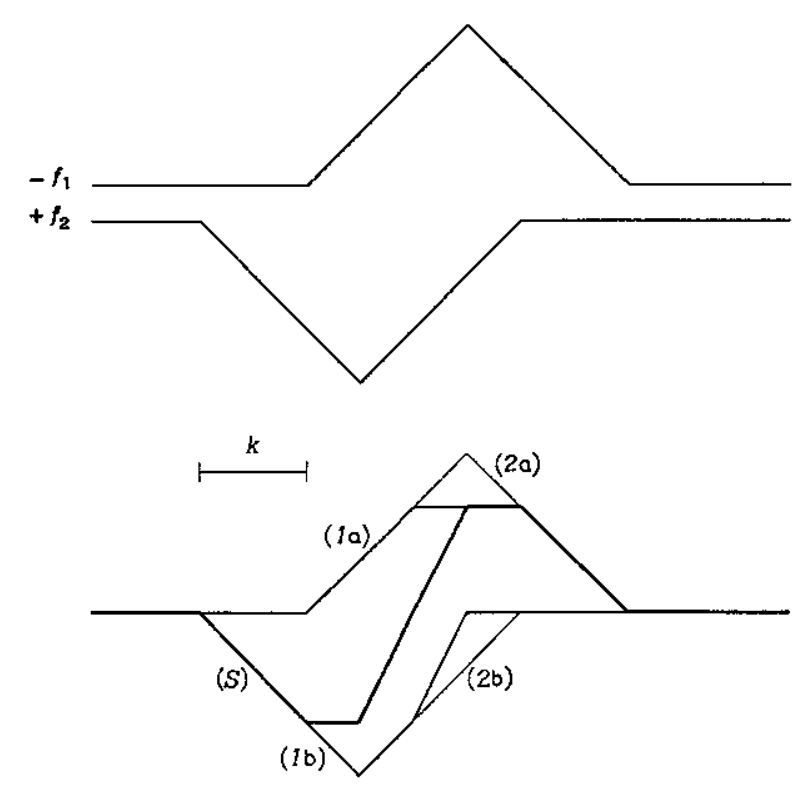

Fig. 2. Reconstruction of a line-profile, drawn schematically as a triangle. Above: the original line images $-f_{1}$ and $f_{2}$, displaced by the shift $k$, which are merged into the $S$-spectrum. Below: from the $S$-spectrum (thick line), the profile restoration is achieved step by step, here in 2 iterations (progressively thinner lines): $(S)=S$-profile; $(1 a)=$ $\max \left\{(S),-(S)_{\mathrm{k}}\right\} ;(1 b)=(S)-(1 a) ;(2 a)=\max \left\{(S),-(1 b)_{\mathrm{k}}\right\}$; $(2 b)=(S)-(2 a)=$ original line-profile $f_{2}$. Note: index $k$ means that the spectrum is red-shifted by $k$

The reconstruction can be carried out in a practical way, by repeatedly comparing the $S$-profile with a shifted and reversed image of itself, which is implemented step by step until the complete line profile is formed. The steps are shown in Fig. 2, considering a schematic triangle-shaped line. Let us remark how easy the procedure is, involving only elementary operations.

The requested number $n$ of steps is proportional to the width of the feature to be reconstructed. Since each iteration restores only a portion of the profile, and the length of this portion is $k$, then:

$$
n=\operatorname{int}\left(\frac{\lambda_{\mathrm{b}}-\lambda_{\mathrm{a}}}{k}\right)+1,
$$

$\left[\lambda_{\mathrm{a}}, \lambda_{\mathrm{b}}\right]$ being the total width of the feature. Normally, the integer $n$ is generally a small number (a few units).

This method can be applied also in the general case when the function $f_{1}(\lambda)$ represents a full set of spectral lines, provided that it still contains one window on the flat continuum, wider than $k$. This interval $\Delta \lambda>k$, in which $f_{1}=0$, is the required basis for running our reconstruction procedure; otherwise, the iterations will not converge.

In practice, working with a real spectrum, we can consider that the profile of an isolated line reaches the continuum, outside a suitable interval around its peak. When dealing with an observational $S$-spectrum, the extremes of our interval can be found, for example, where the far 'tails' of $S$-profile merge into the noise fluctuations of the continuum itself.

\subsection{Restoration of the original spectra}

Owing to the way it has been defined, the restored function $f_{1}(\lambda)$ describes only the shape of the secondary lines, referring to a 'differential' zero-level continuum. Now, a proper renormalization procedure should be established.

The primary spectrum can be isolated, by simply removing the secondary lines from the normalized systemic spectrum $C_{1}$ :

$$
F_{\mathrm{A}}(\lambda)=C_{1}(\lambda)-f_{1}(\lambda) .
$$

Precisely, $F_{\mathrm{A}}$ is the spectrum of the primary star $A$ at the phase $\Phi_{1}$, normalized with respect to the total systemic intensity (that is the $C_{1}$ level).

In order to be consistent with this normalization, the secondary spectrum needs only to be raised back to its original unity-level continuum (formerly the $C_{1}$ level):

$$
F_{\mathrm{B}}(\lambda)=f_{1}(\lambda)+1 .
$$

So, $F_{\mathrm{B}}$ is the spectrum of the star $\mathrm{B}$ at the same phase, normalized to the systemic intensity as well.

Our restoration procedure, if concluded at this point, may already be useful for most practical applications. Let us note, however, that it does not match the standard definition of normalization, which is generally referred to the star's own intensity.

To proceed further, one should know independently the fractional luminosities $h_{\mathrm{A}}$ and $h_{\mathrm{B}}$ of the two stars (relative height of their continua $\left.{ }^{1}\right)$. Assuming for simplicity's sake a small-enough spectral range $\left[\lambda^{\prime}, \lambda^{\prime \prime}\right]$ where the luminosities of the two stars may be considered constant, the two original intensity-normalized spectra are then:

$$
I_{\mathrm{A}}(\lambda)=\left[F_{\mathrm{A}}(\lambda)-h_{\mathrm{B}}\right] / h_{\mathrm{A}} ; \quad I_{\mathrm{B}}(\lambda)=\left[F_{\mathrm{B}}(\lambda)-h_{\mathrm{A}}\right] / h_{\mathrm{B}} .
$$

1 Referring, more precisely, to the average intensity levels (Sect. 2.1), with $h_{\mathrm{A}}+h_{\mathrm{B}}=1$. 
Finally, $I_{\mathrm{A}}(\lambda)$ and $I_{\mathrm{B}}(\lambda)$ should be correctly shifted in $\lambda$, to compensate for the orbital motion.

Such fully-restored spectra may be obtained, in principle, for eclipsing binaries, where $h_{\mathrm{A}}$ and $h_{\mathrm{B}}$ can be accurately measured by occultation spectrophotometry.

The algorithms introduced in this section have been verified by using tests and simulations, applied to artificial line-profiles and modelled binary spectra, which will be shown in Sects. 3.1 and 3.2. Finally, an application to a true binary star will be presented in Sect. 3.3.

\subsection{Quality requirements and implementations}

Since the iterative procedure takes $n$ steps, any spurious difference between the input data will be replicated $n$ times (and possibly amplified) onto the processed spectra. For this reason, preliminary cosmetics is necessary, to correct CCD defects and/or to remove cosmic-ray spikes. Moreover, the two observational spectra must be carefully equalized before processing, in order to compensate for possible normalization discrepancies.

It is also convenient to contain the propagation of noise, by applying the minimum possible number of iterations, on those parts of the spectrum which do not need complex reconstruction. Generally, sharp metallic lines are already restored at the first step, while iterations may only reconstruct the profiles of broader lines (e.g. Balmer series).

Our method, as defined above, restores the function $f_{1}(\lambda)$ by proceeding in the sense of increasing wavelength (that is rightward in Fig. 2). The quality of the extraction can be improved by applying the symmetric procedure, that is by starting from the reversed difference-spectrum $-S(\lambda)$, and using the opposite shift $-k$; so we can reconstruct the function $f_{2}(\lambda)$ (performing a leftward restoration). Thus, we can derive a new version of the primary spectrum $F_{\mathrm{A}}^{\prime}(\lambda)=C_{2}(\lambda)-f_{2}(\lambda)$; and finally by averaging (after shift compensation) the two versions of $F_{\mathrm{A}}$, we may get a primary spectrum with reduced noise and smaller distortions. Similarly, we can obtain another version for the secondary spectrum $F_{\mathrm{B}}^{\prime}(\lambda)=f_{2}(\lambda)+1$, allowing us to compute an improved mean-version also for $F_{\mathrm{B}}$.

Coaddition of multiple exposures, taken at a given phase, may enhance the $S / N$ ratio. In the same way, by employing different pairs of input phases, more $S$-spectra can be obtained, providing independent reconstructions: their average will further improve the $S / N$ value.

On the other side, problems may arise when attempting the restoration of cool stellar spectra crowded with overlapping lines, and having a poorly-defined continuum. Similarly, edge distortions may arise in the presence of truncated line-profiles at the extremes of the spectrum.

In synthesis, although the method may work also under unfavourable conditions, its full power is displayed only when applied to high-quality observational spectra. Such data are actually provided by state-of-the-art astronomi- cal instrumentation, particularly concerning highly stable, sensitive and flawless receptors. Quality requirements will be even more compelling for the profile-analysis algorithm introduced below.

\subsection{Determination of the $k$-shift}

Our separation method uses the secondary shift $k$ as an input parameter (this also happens, incidentally, for other Doppler-based methods mentioned in Sect. 2.1). If some secondary lines are already evident on the input spectra $C_{1}$ and $C_{2}$, the case is trivial since $k$ can be directly measured. On the other side, difficulties may arise when the secondary signal is revealed only after the compensation of primary lines in the $S$-spectrum.

As shown in Fig. 1, only for non-merged $S$-profiles can one take the peak-to-peak distance $s$ as representative of $k$. Generally, for all merging situations, the assumption $k=s$ is misleading. As a result, the reconstructed lines risk becoming stronger than real (tests in Sect. 3.1).

Given the astronomical importance of some situations of merged profiles, we think it is worthwhile studying a way of determining $k$ independently, before performing the spectral reconstruction. Such a derivation of $k$ may be required, for example, in the following cases: $i$ ) when only intermediate phases are available, and the relative Doppler shift may be not large enough to separate, in the $S$-spectrum, the dual images of metallic lines (gaussian approx.); $i$ i) when the secondary star is so faint that its metallic lines are not detectable, and in the $S$-spectrum only overlapping $\mathrm{HI}$ lines are seen (lorentzian approx.).

The shape of the $S$-profile is studied analytically in the appendix A, where a special algorithm is derived. It performs the conversion $s \rightarrow k$ by assuming a gaussian (or lorentzian) approximation for the secondary lines.

As a straightforward result, the determination of $k$ may allow the computation of the masses of the two components A and B of the system. For example, let us suppose that our $S$-spectrum is obtained by difference from two opposite quadrature phases, correctly shifted to compensate for the motion of primary lines; then $k$ corresponds to twice the secondary radial-velocity, plus twice the primary radial-velocity (just compensated for). Precisely, for circular orbits, we have: $k / \lambda=2\left(K_{\mathrm{A}}+K_{\mathrm{B}}\right) / c$, where $K_{\mathrm{A}}$ and $K_{\mathrm{B}}$ are the maximum radial-velocities (barycentric) of the two stars. As $K_{\mathrm{A}}$ is well measured from primary lines, the knowledge of $k$ will then provide a preliminary determination of $K_{\mathrm{B}}$. This will give us the spectroscopic mass-ratio $q=m_{\mathrm{B}} / m_{\mathrm{A}}=K_{\mathrm{A}} / K_{\mathrm{B}}$ which, combined with other known parameters of the system (e. g., see Batten et al. 1989), may finally provide the masses and other absolute elements of the binary star. 


\section{Tests and applications}

The observational material used for the development and the tests of our reconstruction method, and also for its first application, was obtained at the Observatoire de Haute Provence (OHP) in France. The spectra were taken at the $1.52-\mathrm{m}$ telescope by using the Aurélie spectrometer (Gillet et al. 1994) equipped with its mono-dimensional CCD detector (2048-pixel array).

In the tests hereafter, the basic idea is to construct a composite spectrum, made up of two known originals, and then to check on how well the spectral restoration procedure described in Sect. 2 is able to reproduce them separately. A successful performance will then provide support for the application of our method to the real binary star $I Z \mathrm{Per}$, finally revealing a faint secondary spectrum.

\subsection{Reconstruction of artificial profiles}

In order to evaluate the stability of the reconstruction method, with respect to noise fluctuations on the $S$ spectrum, and to possible errors on the value of the shiftparameter $k$, we prepared some analytically-generated line profiles and derived the $S$-features. Then we reconstructed the lines by applying our procedure, and compared the results with the originals.

We used both gaussian and lorentzian functions to create realistic line-profiles, with superimposed poissonian noise produced by a random number generator (Fig. 3). So a large collection of $S$-profiles was obtained, for $k$ values ranging from 0.1 up to 2 times the line FWHM. Then we reconstructed the input lines, by checking different values of $k$ that ranged from 0.5 to 1.5 times its original value. From the above tests we derived the following conclusions: i) the noise is amplified by each iteration; ii) an error on $k$ leads to an error in the intensity of the restored line (if the input value of $k$ is overestimated the restored lines are deeper than real, and vice-versa).

However, from a practical point of view, these effects are tolerable when we do not need too many $(n \lesssim 10)$ iterations for the reconstruction, when the relative error on $k$ is less than $\sim 10 \%$, and when the noise is small, so that $S / N \gtrsim 10$ on the secondary spectrum. Thus, on the observed composite spectra, we should have $S / N \gtrsim 10 / h_{\mathrm{B}}$ where generally the fractional luminosity of the secondary is $h_{\mathrm{B}} \ll 1$.

\subsection{Separation of simulated binary spectra}

For testing the method under typical observational conditions and with real spectra, we used simulated observations. By using a modelling code (Bradstreet 1993), we constructed some fictitious binary systems, each consisting of a close pair of well-known bright stars. Then we simulated the systemic spectrum, by combining and adapting the two known spectra, to reproduce the relative intensities, radial velocities at different phases, rotational line- broadening, noise, etc. Finally, we applied the separation procedure, and checked how accurately the extracted spectra matched the input originals, for a variety of conceivable conditions.
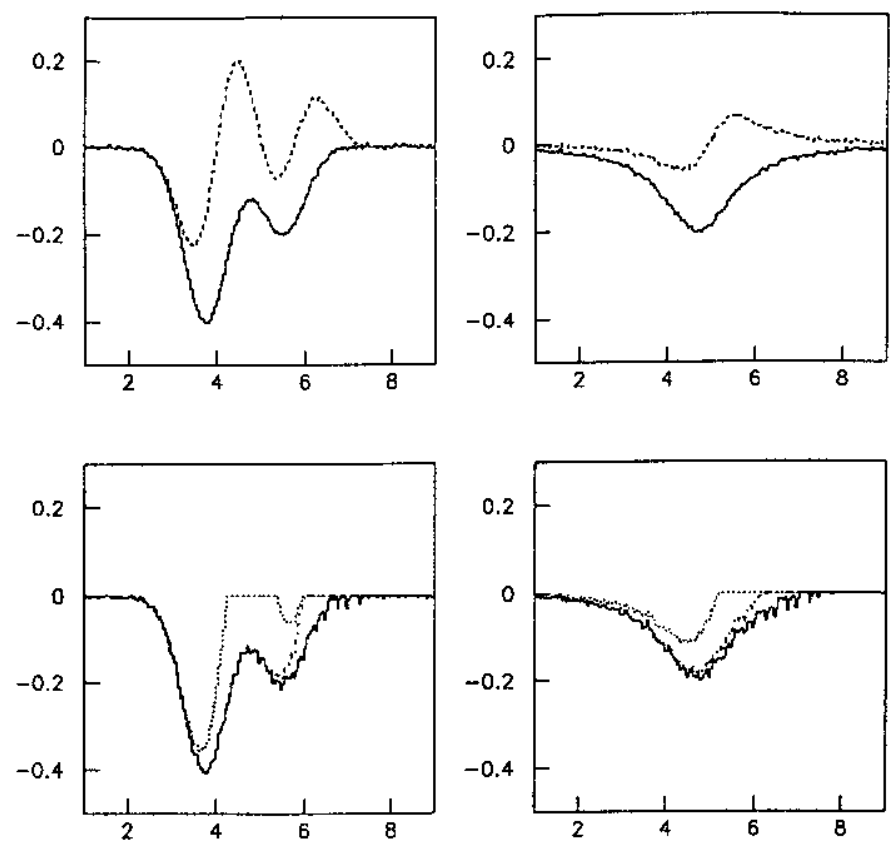

Fig. 3. Tests with artificial profiles: left, gaussian blend; right, lorentzian line. Above: original features (solid), and $S$-spectrum (dashed). Below: step-by-step reconstruction

The spectra used in these simulations (Bédalo 1995) were taken from the Trieste-Aurélie-Archive (TAA). This local facility (Ferluga \& Mangiacapra 1994) contains a collection of high-resolution optical spectra - mainly from standard, peculiar and binary stars - taken at the OHP with Aurélie by observers from Trieste (about 1000 stored spectra). The TAA provides free on-line data retrieval ${ }^{2}$, and it will allow on-line access to our $S$-profile conversion algorithm (Appendix A).

An example of simulation is given by the hypothetical binary EtaVega-2. This object is composed of the pair $A=\eta A u r$ and $B=V e g a$, closely rotating with a supposed period of 2 days. Assuming (for sp. types B3 V+A0 V) the masses $M_{\mathrm{A}}=7.63 M_{\odot}$ and $M_{\mathrm{B}}=2.90 M_{\odot} \quad$ (cf. Schaifers \& Voigt 1981), our model has a separation $a=14.6 R_{\odot}$ between the two stars (while their radii are $R_{\mathrm{A}}=4.80 R_{\odot}$ and $\left.R_{\mathrm{B}}=2.41 R_{\odot}\right)$.

Figure 4 shows the simulated composite spectrum of EtaVega-2, namely $C_{1}$, generated at the first quadrature. By applying the reconstruction procedure, we extracted a secondary spectrum $F_{\mathrm{B}}$. This should be compared with the original spectrum $F_{0}$ of our secondary star (Vega, broadened by $v_{\text {rot }} \simeq 50 \mathrm{~km} \mathrm{~s}^{-1}$ for synchronous rotation).

$\overline{2}$ Soon on the Web (http://www.oat.ts.astro.it). 


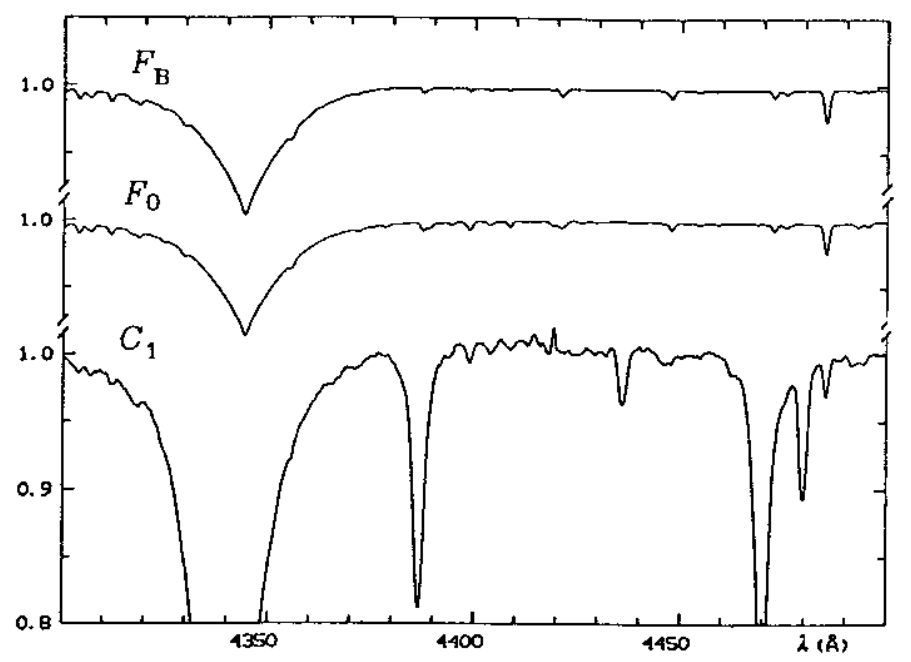

Fig. 4. EtaVega-2, a simulated binary spectrum $\left(C_{1}\right)$. The extracted secondary $F_{\mathrm{B}}$ matches the spectrum $F_{0}$ of $V e g a$

\subsection{An illustrative application to IZ Per}

The earliest idea of our separation technique, to be applied to real astronomical objects, was conceived for the eclipsing binary IZ Per, when we first detected that it was double-lined, and we struggled to isolate the faint secondary spectrum. A pioneering attempt at line reconstruction was made for $\mathrm{H} \gamma$ of IZ Per $B$ (Ferluga et al. 1991).

The actual observations of IZ Per were performed with Aurélie, in the framework of a survey program, in search of double-lined eclipsing binaries (Ferluga et al. 1993). The spectra of the survey, taken at high $S / N$ in the range [4080 $\AA, 4500 \AA]$, are now available in the TAA.

We applied the separation procedure to a pair of mean quadrature spectra, using coadded exposures taken during various orbital cycles. The $k$-parameter for the reconstruction is provided by the $S$-profile of MgII $\lambda 4481 \AA$, where the dual images of the secondary line are split well apart.

Figure 5 displays the resulting spectra of the components. Note the wide $\mathrm{H} \gamma$ wings in the secondary spectrum $F_{\mathrm{B}}$, practically unpredictable by simple visual inspection of the observed systemic spectrum $C_{1}$. The appearing of such feature only in the extracted spectrum is surprising, and one may wonder whether it is definitely real and not an artifact. This is easy to prove. First, the existence of a secondary $\mathrm{H} \gamma$ is revealed by a slight mirror-like asymmetry of the systemic profile at quadratures $C_{1}$ and $C_{2}$ (detectable by careful overplotting). Second, our reconstruction is confirmed by simulations, see EtaVega-2 (Fig. 4) where the extracted $F_{\mathrm{B}}$ perfectly matches the test-profile $F_{0}$ embedded in the composite spectrum $C_{1}$.

For IZ Per $B$, the resulting depth of $\mathrm{H} \gamma$ and $\mathrm{H} \delta$ is about $10 \%$ of the systemic continuum. There are also features of the secondary spectrum reaching only $\sim 1 \%$ of the primary continuum, as the Si II (3) doublet $\lambda \lambda 4128,4131 \AA$ seen just above the noise-level.

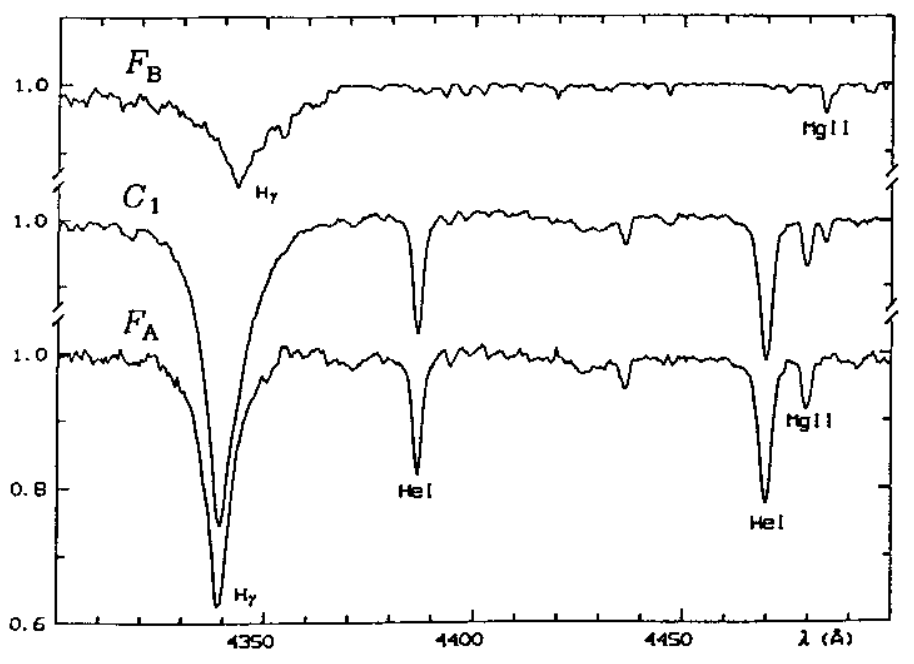

Fig. 5. IZ Per at first quadrature $\left(C_{1}\right)$. The secondary spectrum $F_{\mathrm{B}}$ and the primary spectrum $F_{\mathrm{A}}$ are finally separated

\subsection{Discussion and future work}

Error propagation and causes of scatter, in our procedure and in its implementations (Sect.2.5), were tested experimentally (Sects. 3.1, 3.2) under realistic conditions, with the aim of practical application. a) Spikes and defects in the data strongly disturb the extraction, thus preliminary cosmetics is necessary. b) Normalization discrepancies of the input spectra may cause the algorithm to diverge: this is avoided by rectifying the continuum of the $S$-spectrum. c) Each iteration slightly amplifies the noise, while the two-sided procedure (rightward + leftward) minimizes this effect.

In most cases, the reconstruction is obtained in few iterations (two for IZ Per), simply with the advantage of containing the noise. A favourable situation occurs when many spectra are available at various orbital phases: this means more input pairs to be processed, then more versions of $F_{\mathrm{A}}$ and $F_{\mathrm{B}}$ to be averaged.

Future work will first be devoted to IZ Per itself, and to the application of the separation method to some other eclipsing binaries from the Aurélie survey which show possible secondary lines. Later, application will be extended to other, also non-eclipsing, binary systems.

Finally, we may say that the information provided in this paper is intended to enable anyone who is interested to separate personally his own binary spectra.

Acknowledgements. The authors are indebted to D. Mangiacapra for collaboration in the observations. 


\section{Appendix A: Analysis of the $S$-profile}

Here we shall analyse how information of the secondary component is preserved in the difference-spectrum, and how it can be extracted. In principle, this case is not very different from resolving a blend of two nearby lines, the only peculiarity being that here one component of the blend is in emission, having also the same strength as the other in absorption. So one possible way of studying the difference-spectrum is to consider the $S$-features as being particular blends, treatable by special deblending methods currently available for spectroscopic data-analysis.

The application of a conventional best-fit, however, could not be the optimum choice when the special goal is just to determine $k$. Our idea is that, in a merged $S$-profile, the distortion of the lobes should betray the distance between the two embedded images of the line (while this distortion may be smoothed by fitting). We propose an original method which provides $k$ and the parent-line parameters by directly measuring the shape of the $S$-profile, for gaussian lines (while the lorentzian case is similar).

Let us represent an absorption line of an intensitynormalized spectrum with the gaussian:

$$
F(\lambda)=1-D \cdot \mathrm{e}^{-\pi D^{2}\left(\lambda-\lambda_{0}\right)^{2} / W^{2}},
$$

where $\lambda_{0} D$, and $W$ (central wavelength, central depth, and equivalent width) are the standard parameters of the line. Then the $S$-profile is given by:

$$
S(\lambda)=2 D \cdot \mathrm{e}^{-\pi D^{2}\left[\left(\lambda-\lambda_{0}\right)^{2}+(k / 2)^{2}\right] / W^{2}} \cdot \sinh \frac{\pi D^{2}\left(\lambda-\lambda_{0}\right) k}{W^{2}},
$$

where $k$ is the shift-parameter, as defined above.

The basic parameters of the $S$-profile, which can be directly measured from the difference spectrum, are the following: $\lambda_{1} I_{1}$, and $W_{1}$ (peak wavelength, peak intensity, and equivalent width of the positive lobe), as represented in Fig. 3.4. Alternatively to $\lambda_{1}$, it may be convenient to measure the quantity $s=2\left(\lambda_{1}-\lambda_{0}\right)$, that is the separation between the two $S$-profile peaks.

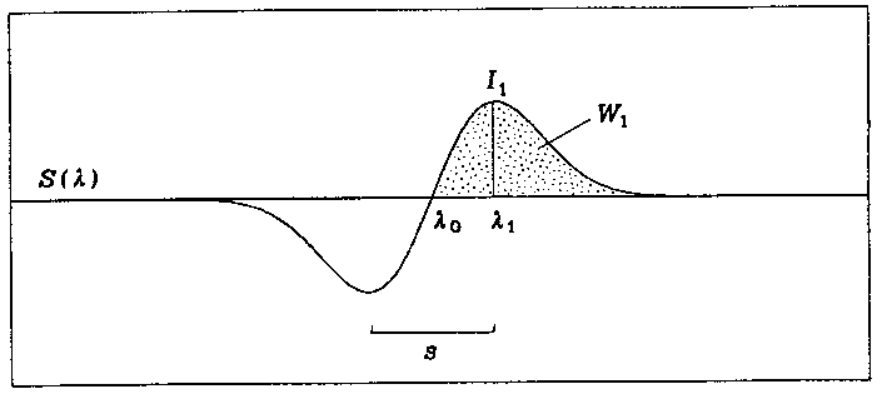

Fig. A1. Basic parameters of the $S$-profile

The peak wavelength $\lambda_{1}$ can be derived, in terms of the original line parameters, from Eq. (A1) with the condition $\mathrm{d} S\left(\lambda_{1}\right) / \mathrm{d} \lambda=0$, leading to:

$$
\frac{k}{s}=\tanh \frac{\pi D^{2} s k}{2 W^{2}}
$$

The other quantity $I_{1}=S\left(\lambda_{1}\right)$ can be also obtained from Eq. (A1) by substitution:

$$
I_{1}=D \cdot\left[\mathrm{e}^{-\pi D^{2}(s-k)^{2} / 4 W^{2}}-\mathrm{e}^{-\pi D^{2}(s+k)^{2} / 4 W^{2}}\right]
$$

and the same can be done for $W_{1}=\int_{\lambda_{0}}^{\infty} S(\lambda) \mathrm{d} \lambda$, which becomes:

$$
W_{1}=D \cdot \int_{-k / 2}^{k / 2} \mathrm{e}^{-\pi D^{2} \lambda^{2} / W^{2}} \mathrm{~d} \lambda
$$

Relations (A2), (A3) and (A4) form a system of implicit equations, where the unknowns are $k, D$, and $W$; while $s, I_{1}$ and $W_{1}$ (parameters of the $S$-profile), together with $\lambda_{0}$ (central wavelength of the $S$-profile), are measurable quantities.

The solution of this system of equations can be achieved by the following half-analytical, half-numerical technique. First, from Eq. (A2) we obtain the term:

$$
\eta(k)=\frac{D^{2}}{W^{2}}=\frac{2}{\pi s k} \operatorname{arctanh} \frac{k}{s} .
$$

Then we substitute this expression in Eq. (A3), writing the central depth as a function of $k$ :

$$
D(k)=I_{1} \cdot\left[\mathrm{e}^{-\pi \eta(k)(s-k)^{2} / 4}-\mathrm{e}^{-\pi \eta(k)(s+k)^{2} / 4}\right]^{-1} .
$$

By substituting the above expressions of $\eta(k)$ and $D(k)$ in Eq. (A4), we finally obtain:

$$
W_{1}=D(k) \cdot \int_{-k / 2}^{k / 2} \mathrm{e}^{-\pi \eta(k) \lambda^{2}} \mathrm{~d} \lambda .
$$

This is an equation in the single unknown $k$; but it still has an implicit form, and is rather complicated. Since it seems impossible to derive the solution analytically, we find the value of $k$ in a numerical form. In fact, there are actually many different routines designed to solve cases such as Eq. (A7).

So, we finally use the resulting value of $k$ to calculate $D$ and $W$ from (A4) and (A5). In conclusion, a practical algorithm can be established (Bravar \& Ferluga 1995), simply making the conversion $\left\{I_{1}, W_{1}, s\right\} \rightarrow\{D, W, k\}$; this will be available within the Trieste-Aurélie-Archive on line (via www).

The above conversion is reliable, as far as the $S$-profile is not remarkably altered by the noise. Only the $S$-features generated by isolated lines can be processed; if more of them are available, a mean value of $k / \lambda$ (same for all lines) can be derived, thus improving the accuracy. 


\section{References}

Bagnuolo W.G., Gies D.R., 1991, ApJ 376, 266

Batten A.H., Fletcher J.M., Mac Carthy D.G., 1989, Eighth Catal. of Orbital Elem. of Spectroscopic Binary Systems, Publ. DAO Victoria (Canada) 17, 1

Bédalo C., 1995, Thesis, Dept. of Astronomy, University of Trieste

Bradstreet D.H., 1993, Binary Maker 2.0, Contact Software 725 Stanbridge, Norristown PA 19401-5505, U.S.A.

Bravar U., Ferluga S., 1995, Internal Rep., Dept. of Astronomy, University of Trieste

Ferluga S., Mangiacapra D., 1991, A\&A 243, 230

Ferluga S., Mangiacapra D., 1993, in: Handling \& Archiving
Data from Ground-based Telescopes, ESO Conf. Rep. 54, 165

Ferluga S., Floreano L., Mangiacapra D., 1991, La Lettre de l' OHP (ed. CNRS, France) 6, 3

Ferluga S., Floreano L., Mangiacapra D., 1993, in: Inside the Stars (IAU Coll. 137), PASPC 40, 364

Gillet D., et al., 1994, A\&AS 108, 1

Griffin R.\& R., 1986, JA\&A (India) 7, 195

Griffin R.E.M., et al., 1993, A\&A 274, 225

Pilachowski C.A., Sowell J.R., 1992, AJ 103, 1668

Schaifers, Voigt, 1981, Landolt-Börnstein VI, Vol.2b: Astron. and Astrophys. Springer Verlag, p. 30

Simon K.P., Sturm E., 1993, A\&A 281, 286

Wright K.O., 1954, Publ. DAO Victoria (Canada) 10, 1 